PROCEEDINGS OF THE

AMERICAN MATHEMATICAL SOCIETY

Volume 140, Number 4, April 2012, Pages 1113-1124

S 0002-9939(2011)11010-4

Article electronically published on November 16, 2011

\title{
FROM PARKING FUNCTIONS TO GELFAND PAIRS
}

\author{
KÜRŞAT AKER AND MAHİR BİLEN CAN
}

(Communicated by Jim Haglund)

\begin{abstract}
A pair $(G, K)$ of a group and its subgroup is called a Gelfand pair if the induced trivial representation of $K$ on $G$ is multiplicity free. Let $\left(a_{j}\right)$ be a sequence of positive integers of length $n$, and let $\left(b_{i}\right)$ be its non-decreasing rearrangement. The sequence $\left(a_{i}\right)$ is called a parking function of length $n$ if $b_{i} \leq i$ for all $i=1, \ldots, n$. In this paper we study certain Gelfand pairs in relation with parking functions. In particular, we find explicit descriptions of the decomposition of the associated induced trivial representations into irreducibles. We obtain and study a new $q$-analogue of the Catalan numbers $\frac{1}{n+1}\left(\begin{array}{c}2 n \\ n\end{array}\right), n \geq 1$.
\end{abstract}

\section{INTRODUCTION}

For a given natural number $n$ and a sequence of positive integers $\left(a_{j}\right)$, let $\left(b_{i}\right)$ denote the non-decreasing rearrangement of $\left(a_{j}\right)$. Then, the sequence $\left(a_{i}\right)$ is called a parking function of length $n$ if $b_{i} \leq i$.

Parking functions, introduced in the papers of Pyke [7, Konheim and Weiss [4] in relation with hashing problems, are related to various topics in algebraic combinatorics: labelled trees, hyperplane arrangements, non-crossing set partitions, diagonal harmonics... . For more, see the solution to problem 5.49 in [9] and the references therein.

A clever argument by Pollak counts the number of parking functions of length $n$ by constructing a one-to-one correspondence between the set of parking functions and the group $\mathbb{Z}_{n+1}^{n} / \Delta \mathbb{Z}_{n+1}^{n}$, where $\mathbb{Z}_{n+1}:=\mathbb{Z} /(n+1) \mathbb{Z}$ denotes the cyclic group of order $n+1$ and $\Delta \mathbb{Z}_{n+1}^{n}$ stands for the diagonal in $\mathbb{Z}_{n+1}^{n}=\mathbb{Z}_{n+1} \times \cdots \times \mathbb{Z}_{n+1}$. This correspondence sends a parking function $\left(a_{i}\right)$ to the element $\left(a_{i}-1 \bmod n+1\right)$. Consequently, the number of parking functions of length $n$ is $P_{n}=(n+1)^{n-1}$.

One can go one step further than Pollak and view the set of parking functions of length $n$ as a finite abelian group. This group is naturally endowed with a symmetric group action by permuting the coordinates. The symmetric group action is compatible with the group operation of the group.

On the representation theory side, this line of inquiry leads us to the realm of Gelfand pairs. On the combinatorics side, it motivates us to define a new $q$-analogue of the Catalan numbers.

Received by the editors February 10, 2010.

2010 Mathematics Subject Classification. Primary 20C30, 05A19, 05E18.

(C)2011 American Mathematical Society

Reverts to public domain 28 years from publication 
1.1. Outline. Let $\Gamma$ be a finite Abelian group, and for each $n \geq 1$ denote by $\widetilde{\Gamma^{n}}$ the quotient of $\Gamma \times \cdots \times \Gamma$ (n copies) by its diagonal subgroup $\Delta \Gamma^{n}:=\{(g, \ldots, g)$ : $g \in \Gamma\}$. Consider $\widetilde{\Gamma^{n}} \rtimes S_{n}$, the semidirect product of $\widetilde{\Gamma^{n}}$ with the symmetric group $S_{n}$.

Our main results are as follows: The pair $\left(\widetilde{\Gamma^{n}} \rtimes S_{n}, S_{n}\right)$ is a Gelfand pair (Lemma 3.2). For finite cyclic groups, $\Gamma=\mathbb{Z}_{r}, r \in \mathbb{N}$ in Theorem 3.5 we find explicit descriptions of the irreducible constituents of the multiplicity-free representation $\operatorname{Ind}_{S_{n}} \widetilde{\Gamma^{n}} \rtimes S_{n}(1)$. Note that when $r=n+1$, the group $\widetilde{\Gamma^{n}}$ is the "group of parking functions", $\mathbb{Z}_{n+1}^{n} / \Delta \mathbb{Z}_{n+1}^{n}$. Let $\bigoplus_{\alpha} V_{\alpha}$ be the decomposition of $\operatorname{Ind}_{S_{n}}^{\widetilde{\Gamma^{n}}} \rtimes S_{n}(1)$ into irreducibles, and let $C_{n}(q)=\sum_{\alpha} q^{\operatorname{dim} V_{\alpha}}$. For $n \in \mathbb{N}$, let

$$
D(n):=\left\{\left(k_{0}, \ldots, k_{n}\right) \in \mathbb{N}^{n+1}: \sum k_{i}=n, \text { and } n+1 \text { divides } \sum_{i=1}^{n} i k_{i}\right\} .
$$

The corollaries of our main result imply that

$$
C_{n}(q)=\sum_{\mathbf{k} \in D(n)} q^{\left(k_{0}, k_{1}, \ldots, k_{n}\right)},
$$

where $\left(\begin{array}{c}n \\ k_{0}, k_{1}, \ldots, k_{n}\end{array}\right)$ is a multinomial coefficient. Hence,

$$
\begin{array}{ll}
C_{n}(1) & =\frac{1}{n+1}\left(\begin{array}{c}
2 n \\
n
\end{array}\right), \text { and } \\
\left.\frac{d C_{n}(q)}{d q}\right|_{q=1} & =(n+1)^{n-1} .
\end{array}
$$

Observe that $D(n)$ can be identified with the set of polynomials $p(x) \in \mathbb{Z}[x]$ with non-negative integer coefficients such that $p(1)=n$, and $p^{\prime}(1)$ is divisible by $n+1$.

Let $S(n)$ denote the set of all integer sequences $\mathbf{b}=\left(b_{1}, \ldots, b_{n}\right) \in \mathbb{N}^{n}$ satisfying $\sum_{i=1}^{j} b_{i} \geq j$ for all $j=1, \ldots, n$, and $\sum_{i=1}^{n} b_{i}=n$. It is well known that $|S(n)|=$ $C_{n}(1)$. See $\left(o^{5}\right)$ in [10. Define

$$
S_{n}(q)=\sum_{\mathbf{b} \in S(n)} q^{\left(b_{1}, \ldots, b_{n}\right)} .
$$

Conjecture 1.1. The polynomials $C_{n}(q)$ and $S_{n}(q)$ are identical for all $n \geq 1$.

The conjecture has been verified for $n \leq 10$ using a computer.

We organized our paper as follows. In Section 2 we introduce our notation and background. In Section 3 we prove our main theorem. In Section 4 we introduce and study a $q$-analogue of the Catalan numbers. We end our article with final remarks and open questions in Section 5 .

\section{Preliminaries}

It is clear from the definition that the symmetric group on $n$ letters $S_{n}$ acts on the set of parking functions of length $n$. For $n=3$, the set of parking functions is divided into 5 orbits:

111

$112,121,211$

$113,131,311$

$122,212,221$

123, 132, 213, 231, 312, 321.

Each line corresponds to an orbit whose first entry is chosen to be non-decreasing. 
Note that orbits can be parameterized in terms of non-decreasing parking functions. Recall that the number of non-decreasing integer sequences $\left(b_{1}, \ldots, b_{n}\right)$ such that $1 \leq b_{i} \leq i$ for all $i=1, \ldots, n$ is the $n$-th Catalan number,

$$
C_{n}:=\frac{1}{n+1}\left(\begin{array}{c}
2 n \\
n
\end{array}\right) \text {. }
$$

2.1. Permutation representation. Given a group $G$ and an action of $G$ on some set $X$, the space of complex-valued functions $\mathrm{E}(X)$ on $X$ becomes a representation of $G$ : Given $g \in G$ and $\psi: X \rightarrow \mathbb{C}$, define the action of $G$ on $\mathrm{L}(X)$ as follows:

$$
(g \cdot \psi)(x):=\psi\left(g^{-1} \cdot x\right) \text {. }
$$

The representation $\mathrm{\textrm {L }}(X)$ induced from the action of $G$ on the set $X$ is called $a$ permutation representation. For any $x \in G$, by $\delta_{x}$ denote the Dirac mass at $x$, defined by

$$
\delta_{x}(y)= \begin{cases}1, & \text { if } x=y \\ 0, & \text { otherwise. }\end{cases}
$$

When $G$ acts on a set $X$, then $X$ is decomposed into $G$-orbits. Similarly, the representation $\mathrm{E}(X)$ is decomposed into irreducible representations. However, by virtue of linearity, the decomposition of $\mathrm{e}(X)$ is much finer than that of the set $X$.

Denote the set of orbits of $X$ by $\operatorname{Orb}(X)$. Each $G$-orbit is stable under the $G$ action; that is, if $x \in O$ for some orbit $O$ and $g \in G$, then $g \cdot x \in O$. Therefore to each orbit we can attach a representation $\mathrm{E}(O)$. Since

$$
X=\bigsqcup_{O \in \operatorname{Orb}(X)} O,
$$

the representation $\mathrm{E}(X)$ decomposes as $\mathrm{\textrm {L }}(X)=\bigoplus_{O \in \operatorname{Orb}(X)} \mathrm{\textrm {L }}(O)$. However, typically one can decompose $\mathrm{E}(X)$ further by decomposing the representations $\mathrm{E}(O)$ corresponding to the orbits $O \in \operatorname{Orb}(X)$. By $|Y|$ denote the number of elements of a finite set $Y$. Then

$$
\operatorname{dim} \mathrm{E}(O)=\text { Number of elements of } O=|O| .
$$

2.2. Parking function module. When $X$ is the set of parking functions of length $n$, we call the representation $\mathrm{E}(X)$ the parking function module and denote it by $\mathrm{PF}(n)$.

Let us go back to the set of parking functions of length 3 . The symmetric group $S_{3}$ acts on the set of parking functions of length 3 and divides it into 5 orbits. We may parameterize the orbits of $S_{n}$ on $\operatorname{PF}(n)$ by the non-decreasing elements they contain. Then,

$$
\mathrm{PF}(3) \cong \mathrm{E}(\mathbf{1 1 1}) \oplus \mathrm{\textrm {L }}(\mathbf{1 1 2}) \oplus \mathrm{\textrm {L }}(\mathbf{1 1 3}) \oplus \mathrm{\textrm {L }}(\mathbf{1 2 2}) \oplus \mathrm{\textrm {L }}(\mathbf{1 2 3}) .
$$

It is well known that $S_{3}$ has 3 irreducible representations: the trivial representation (of dimension 1) which acts trivially on the one-dimensional complex space, the sign representation (of dimension 1) which acts by the sign of the permutation on the one-dimensional complex vector space and a two-dimensional representation.

The symmetric group $S_{3}$ acts on 3 letters $\{1,2,3\}$, hence on the corresponding function space $\mathrm{E}(X)$, called the standard representation. The function $\delta_{1}+\delta_{2}+$ $\delta_{3}$ is invariant under the $S_{3}$-action and forms a one-dimensional representation (isomorphic to the trivial representation). The vector space $V$ is endowed with the standard inner product $\langle\cdot, \cdot\rangle$, where $\left\langle\delta_{i}, \delta_{j}\right\rangle$ is 1 only if $i=j$, and 0 otherwise. Let $V$ 
be the orthogonal subspace to $\delta_{1}+\delta_{2}+\delta_{3}$. Because the inner product is compatible with the $S_{3}$-action, the space $V$ is a two-dimensional representation. In fact, it is irreducible. In the basis $\delta_{1}, \delta_{2}, \delta_{3}$, the subspace $V$ can be expressed as

$$
V=\left\{\sum a_{i} \delta_{i}: \sum a_{i}=0\right\} .
$$

Of the list above, $\mathrm{E}(\mathbf{1 1 1})$ is isomorphic to the trivial representation, the three-

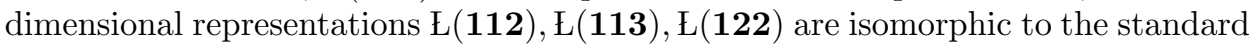
representation (hence decompose as the sum of a trivial representation and the twodimensional representation), and $\mathrm{E}(\mathbf{1 2 3})$ is the regular representation of the group $S_{3}$.

Given a representation $V$ and an irreducible representation $V_{i}$ of a group $G$, the number of times the irreducible representation $V_{i}$ appears in the decomposition of the representation $V$ is called the multiplicity of $V_{i}$ in $V$.

We summarize what we have seen in a table of multiplicities:

\begin{tabular}{lccc} 
& triv & sign & 2-dim \\
\hline $\mathrm{L}(\mathbf{1 1 1})$ & 1 & & \\
$\mathrm{~L}(\mathbf{1 1 2})$ & 1 & & 1 \\
$\mathrm{~L}(\mathbf{1 1 3})$ & 1 & & 1 \\
$\mathrm{~L}(\mathbf{1 2 2})$ & 1 & & 1 \\
$\mathrm{~L}(\mathbf{1 2 3})$ & 1 & 1 & 2
\end{tabular}

Observe that the trivial representation appears in each representation $\mathrm{E}(O)$ only once. In fact, we will later prove an equivalent form of

Lemma 2.1. For all $n \geq 1$, the trivial representation of $S_{n}$ appears in each orbit representation $\mathrm{L}(O)$ in $\mathrm{PF}(n)$ exactly once.

2.3. Intertwining maps and multiplicity-free representations. A representation $V$ of $G$ is called multiplicity free if no irreducible representation of $G$ appears more than once in $V$.

A $\mathbb{C}$-linear map $T: V \rightarrow W$ between two representations $V$ and $W$ of $G$ is called intertwining if

$$
T(g \cdot v)=g \cdot T(v)
$$

for any $g \in G$ and $v \in V$. If $V$ and $W$ are irreducible, then there are two cases:

(1) $V$ and $W$ are non-isomorphic, and $T=0$;

(2) $V$ and $W$ are isomorphic, and $T=\lambda \mathbf{1}_{V}$ for some $\lambda \in \mathbb{C}$.

This is called Schur's Lemma. It follows that if $V=\bigoplus V_{i}^{m_{i}}$, where $m_{i} \in \mathbb{Z}_{\geq 0}$ is the decomposition of $V$ into irreducibles, then the set of intertwining operators on $V$, denoted by $\operatorname{End}_{G}(V)$, is

$$
\operatorname{End}_{G}(V)=\operatorname{End}_{G}\left(\bigoplus V_{i}^{m_{i}}\right)=\bigoplus \operatorname{End}\left(\mathbb{C}^{m_{i}}\right) .
$$

Therefore, $V$ is multiplicity free if and only if $m_{i} \leq 1$ for all $i$ if and only if the algebra $\operatorname{End}_{G}(V)$ is commutative.

2.4. Gelfand pairs. Given a finite group $G$ and a subgroup $K$, the group $G$ acts on the left coset space $G / K$. The pair $(G, K)$ is called a Gelfand pair if the $G$ representation $\mathrm{E}(G / K)$ is multiplicity free.

Given two subgroups $K_{1}$ and $K_{2}$ of $G$, the space of functions $\mathrm{\textrm {L }}\left(K_{1} \backslash G / K_{2}\right)$ on the double coset space $K_{1} \backslash G / K_{2}$ can be identified with the space of $K_{1} \times K_{2}$-invariant 
functions on $G$, consisting of those functions $\psi: G \rightarrow \mathbb{C}$ so that $\psi\left(k_{1} g k_{2}\right)=\psi(g)$ for all $\left(k_{1}, k_{2}\right) \in K_{1} \times K_{2}$ and $g \in G$.

The space of intertwining operators $\operatorname{End}_{G} \mathrm{€}(G / K)$ on $\mathrm{€}(G / K)$ coincides with $\mathrm{E}(K \backslash G / K)$. The algebra $\operatorname{End}_{G} \mathrm{\textrm {L }}(G / K)$ is called the Hecke algebra of the pair $(G, K)$. It follows from Schur's Lemma that the pair $(G, K)$ is a Gelfand pair if and only if its Hecke algebra is commutative.

Lemma 2.1 is equivalent to the following:

Lemma 2.2. The pair $(G, K)$ is a Gelfand pair for $G=\operatorname{PF}(n) \ltimes S_{n}$ and $K=S_{n}$.

Equivalently, the pair $\left(G^{\prime}, K^{\prime}\right)$ is a Gelfand pair, where $G^{\prime}=\mathbb{Z}_{n+1}^{n} \ltimes S_{n}$ and $K^{\prime}=\Delta \mathbb{Z}_{n+1}^{n} \ltimes S_{n}$. Indeed, the symmetric group $S_{n}$ fixes the elements on the diagonal $\Delta \mathbb{Z}_{n+1}$ pointwise; hence this action is trivial, and $K^{\prime} \cong \Delta \mathbb{Z}_{n+1}^{n} \times S_{n}$. The group $G^{\prime}$ is an example of a wreath product of finite groups: $G^{\prime}=\mathbb{Z}_{n+1}^{n} \ltimes S_{n}$ is the wreath product of $\mathbb{Z}_{n+1}^{n}$ by $S_{n}$.

2.5. Wreath products. Let $\Gamma$ be a finite group. The symmetric group $S_{n}$ acts on $\Gamma^{n}$ by permuting the coordinates. Form the semi-direct product $\Gamma^{n} \ltimes S_{n}$. The resulting finite group is called the wreath product of $\Gamma$ by $S_{n}$ and denoted by $\Gamma$ < $S_{n}$. An element of $\Gamma 2 S_{n}$ is denoted by the symbol $\left(g_{1}, \ldots, g_{n} ; \sigma\right)$, where $g_{i} \in \Gamma$ and $\sigma \in S_{n}$. The product of two elements $\left(g_{1}, \ldots, g_{n} ; \sigma\right)$ and $\left(h_{1}, \ldots, h_{n} ; \tau\right)$ is

$$
\left(g_{1}, \ldots, g_{n} ; \sigma\right)\left(h_{1}, \ldots, h_{n} ; \tau\right)=\left(g_{1} h_{\sigma^{-1}(1)}, \ldots, g_{n} h_{\sigma^{-1}(n)} ; \sigma \tau\right) .
$$

We have previously stated that the pair $\left(\mathbb{Z}_{n+1} \prec S_{n}, \Delta \mathbb{Z}_{n+1}^{n} \ltimes S_{n}\right)$ is Gelfand. Inspired by this observation, we can ask for any group $\Gamma$ :

Question. Is $\left(\Gamma<S_{n}, \Delta \Gamma^{n} \times S_{n}\right)$ a Gelfand pair?

When $n=2$, the answer is "yes" for any group $\Gamma$. For $n \geq 3$, the results are mixed. Generally, the answer is "no". We have tested this claim using GAP. Some of the results are:

\begin{tabular}{ccc} 
Group $\Gamma$ & true & false \\
\hline$S_{3}$ & $n \leq 5$ & $n=6$ \\
$A_{4}$ & $n \leq 3$ & $n=4$ \\
$G L\left(2, \mathbb{F}_{3}\right)$ & $n=2$ & $n=3$ \\
$S L\left(3, \mathbb{F}_{2}\right)$ & $n=2$ & $n=3$
\end{tabular}

In general, one can view being a Gelfand pair for $(G, K)$ as a measure of distance between the group $G$ and its subgroup $K$. In some sense, for a Gelfand pair $(G, K)$, the distance between the subgroup $K$ and the whole group $G$ is not that big. In the examples above, as $n$ gets larger, the group $G=\Gamma \imath S_{n}$ gets much larger than the subgroup $K=\Gamma \times S_{n}$. We believe that this is why the answer is typically "no".

\section{Generalized PARKING FUnCtions}

Let $\Gamma$ be a finite group. It is known that the pair $\left(\Gamma \imath S_{n}, S_{n}\right)$ is Gelfand, in the case of a finite cyclic group $\mathbb{Z}_{r}$ by $[$, and in the case of an arbitrary Abelian group $\Gamma$ by $[2]$.

For the rest of the article, let $\Gamma$ be a finite Abelian group. By $\Delta \Gamma^{n}$, denote the diagonal copy of $\Gamma$ in $\Gamma^{n}$, and by $\widetilde{\Gamma^{n}}$ the quotient group $\Gamma^{n} / \Delta \Gamma^{n}$. Finally, the image of an element $g \in \Gamma^{n}$ in $\widetilde{\Gamma^{n}}$ is denoted by $\widetilde{g}$. 
The symmetric group $S_{n}$ acts naturally on the product $\Gamma^{n}$, fixes the diagonal $\Delta \Gamma^{n}$ and hence acts on the quotient group $\Gamma^{n} / \Delta \Gamma^{n}$. Denote the resulting $S_{n^{-}}$ representation by $V_{n}$. There is a natural projection

$$
\begin{aligned}
\pi: \Gamma^{n} \rtimes S_{n} & \rightarrow \widetilde{\Gamma^{n}} \rtimes S_{n} \\
(g, \sigma) & \mapsto(\widetilde{g}, \sigma) .
\end{aligned}
$$

Denote the identity element of the symmetric group $S_{n}$ by $e_{0}$.

Lemma 3.1. The group $\widetilde{\Gamma^{n}} \rtimes S_{n}$ is isomorphic to $\left(\Gamma^{n} \rtimes S_{n}\right) /\left(\Delta \Gamma^{n} \rtimes\left\{e_{0}\right\}\right)$.

Proof. Let $\pi: \Gamma^{n} \rtimes S_{n} \rightarrow \widetilde{\Gamma^{n}} \rtimes S_{n}$ be as in (3.1). It is clear that $\pi$ is surjective. We check that $\pi$ is a homomorphism. Indeed:

$$
\begin{aligned}
\pi((g, \sigma)(h, \tau)) & =\pi((g \sigma(h), \sigma \tau)) \\
& =\widetilde{g \sigma(h)}, \sigma \tau) \\
& =(\widetilde{g}, \sigma)(\widetilde{h}, \tau) .
\end{aligned}
$$

Suppose now that $\pi(g, \sigma)=\left(\widetilde{0}, e_{0}\right)$. Then, $\sigma=e_{0}$ and $g \in \Delta \Gamma^{n}$. In other words, the kernel of $\pi$ is $\Delta \Gamma^{n} \rtimes\left\{e_{0}\right\}$. In particular, $\Delta \Gamma^{n} \rtimes\left\{e_{0}\right\}$ is normal in $\Gamma^{n} \rtimes S_{n}$. Since $\Gamma^{n}$ is Abelian, there is a splitting $\Gamma^{n}=H \cdot \Delta \Gamma^{n}$, where $H \cap \Delta \Gamma^{n}=\{0\}$, and $H$ is isomorphic to $\Gamma^{n} / \Delta \Gamma^{n}$. Since

$$
\left(H \rtimes S_{n}\right) \cdot\left(\Delta \Gamma^{n} \rtimes e_{0}\right)=\left(\Delta \Gamma^{n} \cdot H\right) \rtimes S_{n}=\Gamma^{n} \rtimes S_{n},
$$

it follows that $H \rtimes S_{n} \cong \Gamma^{n} / \Delta \Gamma^{n} \rtimes S_{n}$. In other words, $\Gamma^{n} \rtimes S_{n}$ is the semidirect product of $\Delta \Gamma^{n} \rtimes S_{n}$ and $\widetilde{\Gamma^{n}} \rtimes S_{n}$.

Lemma 3.2. $\left(\widetilde{\Gamma^{n}} \rtimes S_{n}, S_{n}\right)$ is a Gelfand pair.

Proof. This follows from [2].

Lemma 3.2 implies Lemma 2.1 and Lemma 2.2

3.1. $\Gamma$ is a finite cyclic group, $\mathbb{Z}_{r}$. The irreducible representations of the group $\Gamma \curlywedge S_{n}=\mathbb{Z}_{r} \prec S_{n}$ are described as follows (see [1]): Let $\mathbf{k}=\left(k_{0}, \ldots, k_{r-1}\right)$ be an $r$-tuple of non-negative integers, and let $\lambda=\lambda_{\mathbf{k}}=\left(\lambda_{1}, \ldots, \lambda_{\ell}\right)$ be the partition $\left(0^{k_{0}}, 1^{k_{1}}, \ldots,(r-1)^{k_{r-1}}\right) 1$ Suppose

$$
\sum_{i=0}^{r-1} k_{i}=n
$$

Then, $\ell=\ell(\lambda)=n$.

Let $x_{1}, \ldots, x_{n}$ be algebraically independent variables, and let

$$
M(\mathbf{k})=\left\{x_{\sigma(1)}^{\lambda_{1}} x_{\sigma(2)}^{\lambda_{2}} \cdots x_{\sigma(n)}^{\lambda_{n}}: \sigma \in S_{n}, \lambda=\lambda_{\mathbf{k}}\right\} .
$$

We denote by $\mathbb{C} M(\mathbf{k})$ the vector space on $M(\mathbf{k})$. Then, $\Gamma$ ? $S_{n}$ acts on $\mathbb{C} M(\mathbf{k})$ by

$$
(g, \sigma) \cdot f\left(x_{1}, \ldots, x_{n}\right):=f\left(g_{1}^{-1} x_{\sigma(1)}, \ldots, g_{n}^{-1} x_{\sigma(n)}\right),
$$

where $g=\left(g_{1}, \ldots, g_{n}\right) \in \Gamma^{n}, \sigma \in S_{n}$, and $f \in \mathbb{C} M(\mathbf{k})$. It is shown in [1] that $\mathbb{C} M(\mathbf{k})$ is an irreducible $\Gamma 2 S_{n}$-module and all irreducible representations of $\Gamma$ ? $S_{n}$ can be obtained this way.

\footnotetext{
${ }^{1}$ In this notation, $k_{i}$ is the multiplicity of $i$ in the partition $\lambda$.
} 
In [6] it is shown that

$$
\operatorname{Ind}_{S_{n}}^{\Gamma 2 S_{n}}(1)=\bigoplus_{\sum k_{i}=n} \mathbb{C} M(\mathbf{k})
$$

Remark 3.3. Let $(g, \sigma) \in \Gamma 2 S_{n}$ and $x_{\tau(1)}^{\lambda_{1}} \cdots x_{\tau(n)}^{\lambda_{n}} \in M(\mathbf{k})$. Then,

$$
\begin{aligned}
(g, \sigma) \cdot\left(x_{\tau(1)}^{\lambda_{1}} \cdots x_{\tau(n)}^{\lambda_{n}}\right) & =(g, \sigma) \cdot\left(x_{1}^{\lambda_{\tau-1}(1)} \cdots x_{n}^{\lambda_{\tau-1}(n)}\right) \\
& =\frac{1}{\prod_{i} g_{\sigma(i)}^{\lambda_{\tau-1}(i)}} x_{\sigma(1)}^{\lambda_{\tau-1}(1)} \cdots x_{\sigma(n)}^{\lambda_{\tau-1}(n)} \\
& =\frac{1}{\prod_{i} g_{\sigma(\tau(i))}^{\lambda_{i}}} x_{\sigma(\tau(1))}^{\lambda_{1}} \cdots x_{\sigma(\tau(n))}^{\lambda_{n}} .
\end{aligned}
$$

Therefore, the matrix of the representation $\mathbb{C} M(\mathbf{k})$ at $(g, \sigma) \in \Gamma$ २ $S_{n}$ is a monomial matrix and its unique non-zero diagonal entry is $1 / \prod_{i} g_{\sigma(i)}^{\lambda_{i}}$. It follows that the character $\chi_{\mathbb{C} M(\mathbf{k})}$ of $\mathbb{C} M(\mathbf{k})$ evaluated at $(g, \sigma)$ equals

$$
\chi_{\mathbb{C} M(\mathbf{k})}(g, \sigma)=\frac{1}{g_{\sigma(1)}^{\lambda_{1}} \cdots g_{\sigma(n)}^{\lambda_{n}}} .
$$

3.2. Spherical functions. Let $(G, K)$ be a Gelfand pair. Suppose that $\bigoplus_{i=1}^{s} V_{i}$ is the decomposition of $\operatorname{Ind}_{K}^{G}(1)$ into irreducible subrepresentations. For $1 \leq i \leq s$, let $\chi_{i}$ denote the character of $V_{i}$ and define the $\mathbb{C}$-valued function

$$
\omega_{i}(x)=\frac{1}{|K|} \sum_{h \in K} \chi_{i}\left(x^{-1} h\right), \text { for } x \in G .
$$

The functions $\omega_{i}$ for $i=1, \ldots, s$ are called the zonal spherical functions of the pair $(G, K)$. They form an orthogonal basis of $\operatorname{End}_{G} \mathrm{E}(G / K)$.

Let $m_{\lambda}\left(x_{1}, \ldots, x_{n}\right)$ be the monomial symmetric function defined as

$$
m_{\lambda}\left(x_{1}, \ldots, x_{n}\right)=\frac{1}{k_{0} ! k_{1} ! \cdots k_{n} !} \sum_{\sigma \in S_{n}} x_{\sigma(1)}^{\lambda_{1}} \cdots x_{\sigma(n)}^{\lambda_{n}} .
$$

In [6], it shown that the zonal spherical function $\omega^{\mathbf{k}}$ corresponding to an irreducible constituent $\mathbb{C} M(\mathbf{k})$ of $\operatorname{Ind}_{S_{n}}^{\Gamma l S_{n}}(1)$ can be expressed in terms of the monomial symmetric function $m_{\lambda}$,

$$
\omega^{\mathbf{k}}((h, \sigma))=\frac{m_{\lambda}\left(h_{1}, \ldots, h_{n}\right)}{m_{\lambda}(1, \ldots, 1)}, \text { for }(h, \sigma) \in \Gamma 2 S_{n},
$$

where $\lambda$ is the partition determined by $\mathbf{k}$.

Remark 3.4. It is easy to see that $m_{\lambda}(1, \ldots, 1)=\left(\begin{array}{c}n \\ k_{1}, \ldots, k_{n}\end{array}\right)$.

3.3. Zonal spherical functions for $\left(\widetilde{\Gamma} n \rtimes S_{n}, S_{n}\right)$. In the rest of the article, we will concentrate on the generalized parking function module

$$
\operatorname{Ind}_{S_{n}}^{\widetilde{\Gamma^{n}}} S_{n}(1) .
$$

We start with a general observation. 
Fact 3.4 (See Section 1.13, Exercise 6.b in [8]). Let $N$ be a normal subgroup of a finite group $G$, and let $Y$ be a representation of $G / N$. Define a function $X$ on $G$ by $X(g)=Y(g N)$. Then, $X$ is an irreducible representation of $G$ if and only if $Y$ is an irreducible representation of $G / N$. In this case $X$ is said to be "lifted from $Y^{\prime \prime}$.

Since $\widetilde{\Gamma^{n}} \rtimes S_{n}$ is isomorphic to $\left(\Gamma \nmid S_{n}\right) /\left(\Delta \Gamma^{n} \rtimes\left\{e_{0}\right\}\right)$, we can use Fact 3.4 Let $\mathbb{C} M(\mathbf{k})$ be an irreducible representation of $\Gamma$ $S_{n}$ for some $\mathbf{k}=\left(k_{0}, \ldots, k_{r-1}\right) \in \mathbb{Z}_{>0}^{r}$, where $\sum k_{i}=n$. The representation $\mathbb{C} M(\mathbf{k})$ descends to a representation $Y$ of $\widetilde{\Gamma^{n}}$ if and only if the normal subgroup $\Delta \Gamma^{n} \rtimes\left\{e_{0}\right\}$ acts trivially on the representation $\mathbb{C} M(\mathbf{k})$, in which case, the representation $Y$ is irreducible by the above fact.

Let $\left(c, e_{0}\right) \in \Delta \Gamma^{n} \rtimes\left\{e_{0}\right\}$ and $x_{\tau(1)}^{\lambda_{1}} \cdots x_{\tau(n)}^{\lambda_{n}} \in M(\mathbf{k})$, where $\xi=e^{\frac{2 \pi i}{r}}$ and $c=$ $\left(\xi^{j}, \ldots, \xi^{j}\right)$ for some $j=0, \ldots, r-1$.

We calculate the effect of $\left(c, e_{0}\right) \in \Delta \Gamma^{n} \rtimes\left\{e_{0}\right\}$ on $x_{\tau(1)}^{\lambda_{1}} \cdots x_{\tau(n)}^{\lambda_{n}}$ :

$$
\left(c, e_{0}\right) \cdot x_{\tau(1)}^{\lambda_{1}} \cdots x_{\tau(n)}^{\lambda_{n}}=\left(\xi^{-j \sum \lambda_{i}}\right) x_{\tau(1)}^{\lambda_{1}} \cdots x_{\tau(n)}^{\lambda_{n}} .
$$

The action of $\Delta \Gamma^{n} \rtimes\left\{e_{0}\right\}$ on $\mathbb{C} M(\mathbf{k})$ is trivial if and only if the right-hand side equals $x_{\tau(1)}^{\lambda_{1}} \cdots x_{\tau(n)}^{\lambda_{n}}$. Given that $\xi$ is a primitive $r$-th root of unity, this is possible if and only if $r$ divides the sum $\sum \lambda_{i}=\sum i k_{i}$.

Thus, $\mathbb{C} M(\mathbf{k})$ is an irreducible representation of $\widetilde{\Gamma^{n}} \rtimes S_{n}$ if and only if $\mathbf{k}=$ $\left(k_{0}, \ldots, k_{r-1}\right)$ satisfies the following two conditions:

- $\sum_{i} k_{i}=n$,

- $r$ divides $\sum i k_{i}$.

Our main result follows from these observations:

Theorem 3.5. Let $\Gamma=\mathbb{Z}_{r}$ and $\mathbf{k}=\left(k_{0}, \ldots, k_{r-1}\right)$ be a sequence of non-negative integers of length $r$. Then,

(1) The $\widetilde{\Gamma^{n}} \rtimes S_{n}$-representation $\mathbb{C} M(\mathbf{k})$ is irreducible of degree $\left(\begin{array}{c}n \\ k_{0}, \ldots, k_{r-1}\end{array}\right)$ if and only if $\sum_{i} k_{i}=n$ and $r$ divides $\sum i k_{i}$.

(2) The pair $\left(\widetilde{\Gamma^{n}} \rtimes S_{n}, S_{n}\right)$ is Gelfand. Furthermore, Ind $d_{S_{n}}^{\widetilde{\Gamma^{n}}} \rtimes_{n}(1)$ decomposes into irreducibles as follows:

$$
\operatorname{Ind} d_{S_{n}}^{\widetilde{\Gamma^{n}}} \rtimes S_{n}(1)=\underset{\substack{\sum k_{i}=n \\ r \mid \sum i k_{i}}}{\bigoplus} \mathbb{C} M(\mathbf{k}) .
$$

Remark 3.6. Let $g \in \Gamma^{n}$ and let $\widetilde{g}$ be its image in $\widetilde{\Gamma^{n}}$. A simple calculation shows that the double coset $S_{n}\left(\widetilde{g}, e_{0}\right) S_{n}$ in $\widetilde{\Gamma^{n}} \rtimes S_{n}$ is $\left\{(\sigma(\widetilde{g}), \sigma \tau): \sigma, \tau \in S_{n}\right\}$. Therefore $\left|S_{n}\left(\widetilde{g}, e_{0}\right) S_{n}\right|$ is the cardinality of the $S_{n}$-orbit $S_{n} \cdot \widetilde{g}$ in $\widetilde{\Gamma}^{n}$ times $n$ !. In addition, the number of double cosets of $S_{n}$ in $\widetilde{\Gamma^{n}} \rtimes S_{n}$ is the number of $S_{n}$-orbits in $\widetilde{\Gamma^{n}}$.

Corollary 3.7. For any positive integers $n$ and $r$ the following identity holds:

$$
r^{n-1}=\sum\left(\begin{array}{c}
n \\
k_{0}, \ldots, k_{r-1}
\end{array}\right)
$$

where the sum runs over all non-negative integer sequences of length $r$ such that $\sum_{i} k_{i}=n$ and $r$ divides $\sum_{i} i k_{i}$. 


\section{A $q$-ANAlog OF the CATAlan Numbers}

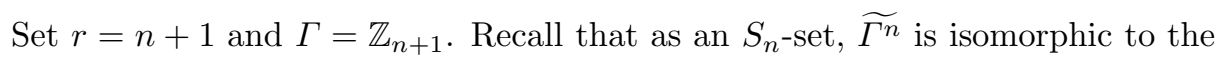
set of parking functions. Recall also that

Fact 4.1. The number of $S_{n}$-orbits in an $S_{n}$-set $X$ is the multiplicity of the trivial representation in the associated representation $\mathrm{E}(X)$.

Proposition 4.1. Let $\Gamma=\mathbb{Z}_{n+1}$. Then, the number of irreducible representations in

$$
\operatorname{Ind}_{S_{n}} \widetilde{\Gamma}_{n} \rtimes S_{n}(1)
$$

is the $n$-th Catalan number, $C_{n}$.

Proof. This is immediate from Fact 4.1

Let $\bigoplus_{\alpha} V_{\alpha}$ be the decomposition of $\operatorname{Ind}_{S_{n}}^{\widetilde{T_{n}^{n}}} S_{S_{n}}(1)$ as in (3.5), where $r=n+1$. Define

$$
C_{n}(q)=\sum_{\alpha} q^{\operatorname{dim} V_{\alpha}}
$$

Since the multiplicity of each irreducible representation in $\operatorname{Ind}_{S_{n}}^{\widetilde{T_{n}}} \rtimes S_{n}(1)$ is 1 , the coefficients of the polynomial $C_{n}(q)$ count the number of occurrences of the irreducible representations of a given dimension in $\operatorname{Ind}_{S_{n}}^{\widetilde{T_{n}^{n}} \rtimes S_{n}}(1)$.

Let $D(n)$ denote the set of all $n+1$ tuples $\mathbf{k}=\left(k_{0}, \ldots, k_{n}\right) \in \mathbb{N}^{n+1}$ such that $\sum_{i=0}^{n} k_{i}=n$ and $n+1$ divides $\sum_{i=1}^{n} i k_{i}$. It follows from Theorem 3.5 that

$$
C_{n}(q)=\sum_{\mathbf{k} \in D(n)} q^{\left(k_{0}, k_{1}, \ldots, k_{n}\right)} .
$$

It follows from Proposition 4.1 that

$$
C_{n}(1)=C_{n}=\frac{1}{n+1}\left(\begin{array}{c}
2 n \\
n
\end{array}\right)
$$

and that

$$
\left.\frac{d C_{n}(q)}{d q}\right|_{q=1}=(n+1)^{n-1} .
$$

Example 4.6. There are 5 sequences of the form $\left(k_{0}, \ldots, k_{3}\right) \in \mathbb{N}^{4}$ such that $k_{0}+\cdots+k_{3}=3$ and $\sum_{i=1}^{3} i k_{i}$ is divisible by 4 . Namely,

$$
D(3)=\{3000,1101,0210,1020,0012\} .
$$

Then $C_{3}(q)=q+3 q^{3}+q^{6}$. Similarly, one can compute $C_{4}(q)=q+4 q^{4}+2 q^{6}+$ $6 q^{12}+q^{24}$.

Let $E(n)$ be the set of $n$-element multisets on $\mathbb{Z}_{n+1}$ whose elements sum to 0 . It is known that the cardinality $E(n)$ is equal to the $n$-th Catalan number. See jjj on page 264 of $[9$. Recently Tewodros Amdeberhan constructed an explicit bijection between the sets $D(n)$ and $E(n)$. 
Set $C_{n}(q)=\alpha_{1}(n) q^{a_{1}}+\alpha_{2}(n) q^{a_{2}}+\cdots+\alpha_{m}(n) q^{a_{m}}$, where $\alpha_{i}(n) \neq 0$ for $i=$ $1, \ldots, m$ and $1<a_{1}<a_{2}<\cdots<a_{m}$. It is desirable to find a combinatorial interpretation of the coefficients $\alpha_{i}(n), 1 \leq i \leq m$.

4.1. Labeled rooted trees. Let us point out an intriguing relationship between the coefficients of $C_{n}(q)$ and the Lagrange inversion formula.

Let $t_{0}<t_{1}<t_{2}<\cdots$ be a collection of ordered, commuting variables. Let $\sigma$ be a plane tree (connected graph with no cycles) on $n$ vertices. Set

$$
t^{\sigma}=\prod_{i \geq 0} t_{i}^{d_{i}(\sigma)}
$$

where $d_{i}(\sigma)$ is the number of vertices of $\sigma$ of degree $i$. Define

$$
s_{n}=\sum_{\tau} t^{\tau}
$$

summed over all plane trees with $n$ vertices. For example, $s_{4}=t_{0}^{3} t_{3}+3 t_{0}^{2} t_{1} t_{2}+t_{0} t_{1}^{3}$. Using lexicographic ordering on the monomials of $s_{n}$, we denote by $v_{n}$ the sequence of coefficients of the polynomial $s_{n}$. For example, $v_{4}=(1,3,1)$. It turns out, for $n \leq 6$, that the sequence $v_{n+1}$ agrees with the sequence $\left(\alpha_{1}(n), \ldots, \alpha_{m}(n)\right)$ of coefficients of $C_{n}(q)$. However,

$$
\left(\alpha_{i}(7)\right)_{i=1}^{14}=(1,7,7,7,21,42,21,56,105,35,35,70,21,1),
$$

and $v_{8}=(1,7,7,7,21,42,21,21,35,105,35,35,70,21,1)$.

It is well known that the coefficient vectors $v_{n}, n \geq 1$ can be computed by the Lagrange inversion formula (see page 40 of [9]).

\section{Final Remarks AND QUeStions}

Let $\mathbf{k}=\left(k_{0}, \ldots, k_{n}\right)$ be an element of $D(n)$ and let $\mathbb{C} M(\mathbf{k})$ be the associated irreducible constituent of $\operatorname{Ind}_{S_{n}}^{\widetilde{\Gamma^{n}}} \bigwedge_{n}(1)$. Let $\omega_{P F}^{\mathbf{k}}$ be the zonal spherical function.

Given $(h, \sigma) \in\left(\Gamma^{n}\left\{S_{n}, S_{n}\right)\right.$, by $\widetilde{h}$ denote the image of $h$ in $\widetilde{\Gamma^{n}}$. Then,

$$
\omega_{P F}^{\mathbf{k}}(\widetilde{h}, \sigma)=\frac{m_{\lambda}\left(h_{1}, \ldots, h_{n}\right)}{m_{\lambda}(1, \ldots, 1)}
$$

where $\lambda$ is the partition determined by $\mathbf{k} \in D(n)$.

The values of the zonal spherical functions for $\widetilde{\Gamma^{n}} \rtimes S_{n}$ are not necessarily real. For instance, the zonal spherical function $\omega^{\mathbf{k}}: \widetilde{\Gamma^{5}} \rtimes S_{5} \rightarrow \mathbb{C}$ for $\mathbf{k}=(0,0,0,2,3)$, depicted in Figure 1, is not real valued.

What about the number of real-valued zonal spherical functions? A few terms of this sequence are $2,3,6,10, \ldots$ (starting at $n \geq 2$ ). Can we say anything about the injectivity of these functions? How about their Fourier transforms?

Let $\left\{x_{1}, y_{1}, \ldots, x_{n}, y_{n}\right\}$ be a set of $2 n$ algebraically independent variables on which $\sigma \in S_{n}$ acts by

$$
\sigma \cdot x_{i}=x_{\sigma^{-1}(i)}, \sigma \cdot y_{i}=y_{\sigma^{-1}(i)} \text { for } i=1, \ldots, n .
$$




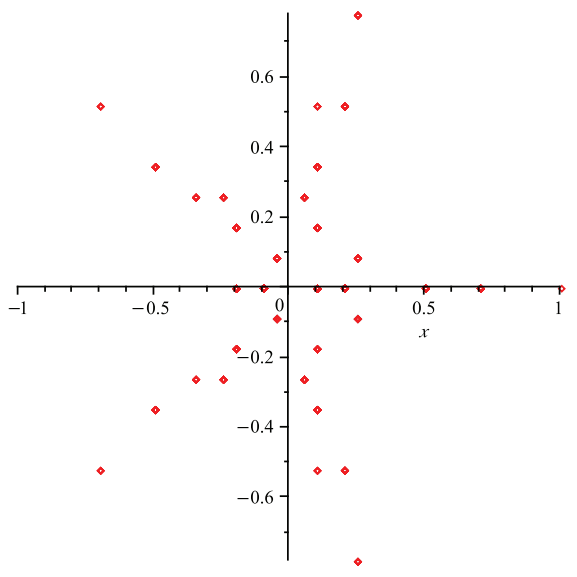

FiguRE 1

For non-negative integers $r, s \in \mathbb{N}$, define $p_{r, s}=\sum_{i=1}^{n} x_{i}^{r} y_{i}^{s}$. The ring of diagonal co-invariants (of $S_{n}$ ) is the $S_{n}$-module

$$
R_{n}=\mathbb{C}\left[x_{1}, y_{1}, \ldots, x_{n}, y_{n}\right] / I_{+},
$$

where $I_{+}$is the ideal generated by the power sums $p_{r, s}$ with $r+s>0$.

Let $\Gamma=\mathbb{Z}_{n+1}$. It is known that as an $S_{n}$-module, $R_{n}$ is isomorphic to $\left(\Gamma^{n} / \Delta \Gamma^{n}\right) \otimes \operatorname{sign}($ see [3] $)$. Notice that $R_{n}$ is a bi-graded $S_{n}$-module. Let $\mathcal{F}(q, t)$ be the corresponding bi-graded character. The multiplicity of the sign character in $\mathcal{F}(q, t)$ is the so-called $q, t-$ Catalan series. What is the relationship between the $q, t$-Catalan series and our $q$-analogue? It would be interesting to also investigate similar questions for the associated "twisted Gelfand pair". (For a definition, see [5], page 398.)

\section{ACKNOWLEDGEMENT}

The authors thank Tewodros Amdeberhan for helpful discussions on the Catalan items.

\section{REFERENCES}

[1] Susumu Ariki, Tomohide Terasoma, and Hiro-Fumi Yamada. Higher Specht polynomials. Hiroshima Math. J., 27(1):177-188, 1997. MR1437932 (98c:05163)

[2] Tullio Ceccherini-Silberstein, Fabio Scarabotti, and Filippo Tolli. Trees, wreath products and finite Gelfand pairs. Adv. Math., 206(2):503-537, 2006. MR2263713 (2007k:43017)

[3] Mark D. Haiman. Conjectures on the quotient ring by diagonal invariants. J. Algebraic Combin., 3(1):17-76, 1994. MR.1256101 (95a:20014)

[4] Alan G. Konheim and Benjamin Weiss. An occupancy discipline and applications. SIAM J. Applied Math., 14:1266-1274, 1966.

[5] I. G. Macdonald. Symmetric functions and Hall polynomials. The Clarendon Press, Oxford University Press, New York, 1979. Oxford Mathematical Monographs. MR 553598|(84g:05003)

[6] Hiroshi Mizukawa. Zonal spherical functions on the complex reflection groups and $(n+1$, $m+1)$-hypergeometric functions. Adv. Math., 184(1):1-17, 2004. MR2047846 (2005f:33016)

[7] Ronald Pyke. The supremum and infimum of the Poisson process. Ann. Math. Statist., 30:568-576, 1959. MR0107315 (21:6040) 
[8] Bruce E. Sagan. The symmetric group, volume 203 of Graduate Texts in Mathematics. Representations, combinatorial algorithms, and symmetric functions, Springer-Verlag, New York, second edition, 2001. MR.1824028 (2001m:05261)

[9] Richard P. Stanley. Enumerative combinatorics. Vol. 2, volume 62 of Cambridge Studies in Advanced Mathematics. Cambridge University Press, Cambridge, 1999. With a foreword by Gian-Carlo Rota and appendix 1 by Sergey Fomin. MR.1676282 (2000k:05026)

[10] Richard P. Stanley. Catalan Addendum. http://www.math.mit.edu/ ${ }^{\sim}$ rstan/ec/catadd.pdf, 2010.

Feza Gürsey Institute, Istanbul, Turkey

E-mail address: aker@gursey.gov.tr

Tulane University, New Orleans, Louisiana 70118

E-mail address: mcan@tulane.edu 